\title{
Thermoelastic stress profiles for different frequencies
}

\author{
by António Ramos Silva ${ }^{a, b, c}$, Mário Vaz ${ }^{a, c}$, P. Moreira ${ }^{c}$, Sofia Ribeirinho Leite ${ }^{\text {b, d }}$ and Joaquim \\ Mendes ${ }^{\text {a, c, e }}$
}
a) FEUP - Universidade do Porto, Faculdade de Engenharia, Rua Dr. Roberto Frias, s/n 4200-465 Porto
PORTUGAL, a.ramos@fe.up.pt;
b) Dare Institute, Cambridge, MA, USA
c) INEGI - Institute of Science and Innovation in Mechanical and Industrial Engineering, Rua Dr. Roberto Frias, Campus da FEUP, 400, Porto, Portugal;
d) CINTESIS - Center for Health Technology and Services Research, Faculty of Medicine, University of Porto
e) LABIOMEP - Porto Biomechanics Laboratory, Rua Dr. Plácido Costa 91, Porto, Portugal;

\section{Introduction}

Nowadays, structural components where weight is a critical requirement, like in aeronautics, are usually designed based on a damage tolerance principle. This leads to a near $90 \%$ of the failures by crack and fatigues. [1]. The early detection of cracks is of the utmost importance, both for the structure and users. One of the best solutions is to use nondestructive image testing techniques to locate cracks prematurely, preventing serious damages [2]. The usage of Thermal testing to access the stress in a component is a well-established technique. Here, a sinusoidal load is applied and the thermal amplitude is calculated, which is then correlated with the mechanical stress. Using the model developed by D. S. Mountain and J. M. B. Webber (1979) and their thermoelastic stress analyses (TSA) system, the SPATE [3]. According to the literature, a load should be applied with a frequency between 5 and $200 \mathrm{~Hz}$ [4].

The main goal of this work was to access the influence of the stimulation frequency in the thermal patterns during a thermal stress test/analysis (TSA). A detailed emphasis is given to the crack tip. The tests and consequent analyses were performed for several frequencies in order to assess its influence in the thermal patterns.

\section{Methods}

\subsection{Thermoelastic Stress Tests}

The specimen was made of aluminum, two millimeters thick (2219-T851), with the geometry following the recommendation of the ASTM E 647-08 for a compact specimen. This was painted with a black mate black varnish (kameralack) for a higher and uniform emissivity.

To apply the load an Instron ${ }^{\circ}$, model ElectroPuls E1000 was used, applying sinusoidal loads ranging from 75 to $750 \mathrm{~N}$ with a sinusoidal profile, at a constant frequency. The thermal camera was from FLIR ${ }^{\text {TM }}$, model 7500 MWIR with a measurement waveband from 1.5 to $5.1 \mu \mathrm{m}$ and a spatial resolution of $320 \times 256$ pixels. In all tests, the acquisition frequency was adjusted to ten times higher than the stimulation frequency.

\subsection{Procedure}

The crack length was measured at the beginning and end of the two sets of TSA tests. Each measurement consisted of 1050 cycles for the crack progression and 200 cycles for measurement (a TSA test).

The specimen tests were divided into various sets, with a frequency of: $2.5,5,10,20,30,40,50,60$, and $70 \mathrm{~Hz}$ at the monitorization phase (250 cycles).

The processing algorithm used to assess the stress images is based on the lock-in principle. It uses a reference signal from the test machine as a reference input (100 N/V) to calculate the amplitude and phase response of each pixel. The literature suggests approximately $8.2 \mathrm{E}-6$ for the aluminum thermoelastic coefficient, however, the software manual (IRNDT version 1.3) indicates the value of $7.7 \mathrm{E}-6$. This last was confirmed using a rectangular aluminum bar with $180 \mathrm{x}$ $10 \times 1.5 \mathrm{~mm}$ with two strain gages, one on each side. This measured strain was then compared with the obtained using the TSA method.

\section{Results}

This work assesses the impact of different load frequencies in TSA tests. Thus, the central mechanical stress profiles for the selected frequencies were analyzed and compared (figure 1). Near the crack tip, and in a small region, stress values vary considerably among themselves. A low pass filter was applied to the TSA results, thus achieving a better overall comparison. After a certain value, the noise in the phase image is irrelevant and therefore the only noise visible in the stress image is resulting from the thermal amplitude image. 
All the tests revealed that a higher load frequency resulted in a higher maximum stress peak. The peak mechanical stress location represents the location where the temperature amplitude was also higher. Observing the stress profiles obtained with TSA, a relation between these and the loading frequencies is observed.

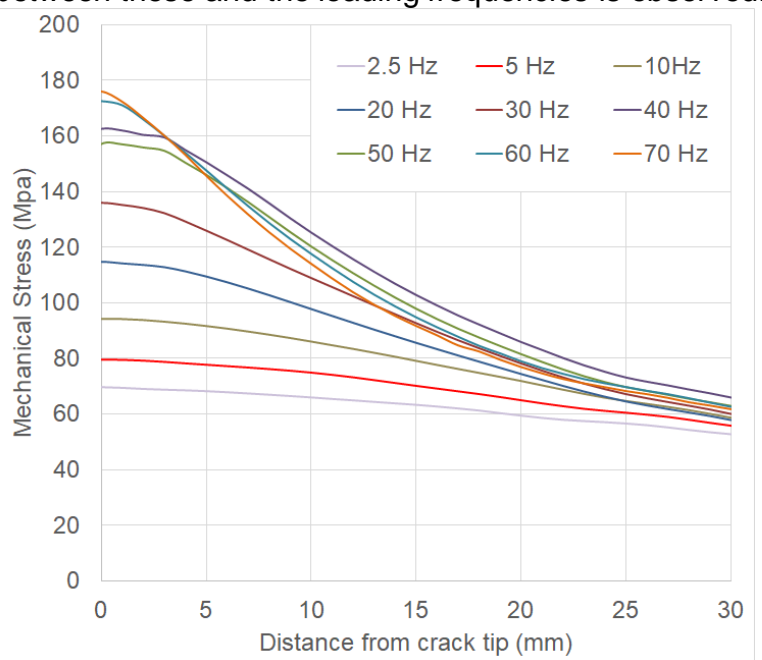

Fig. 1. Mechanical stress curves obtained for different frequencies.

\section{Discussion}

Due to the increase of the stress concentration factor, a very small growth of the thermal profile was expected at each test and mainly from the first to the second phase. This was clearly observed in the specimen tested up until rupture with a progression of approximately 1250 cycles between measurements. For each measurement, 1050 cycles for the crack progression and 200 cycles for TSA measurement were used. However, for the specimen tested with different frequencies, these observations were somewhat different, especially when blindly comparing the results of the two phases. By comparing the profiles, a crack progression of near 0.3 millimeters is estimated.

When analyzing figure 1, it is clear that the increase in the stress peak from the lower frequencies to the higher is not due to the increment of the crack progression and the correspondent concentration factor. This is a clear indication that the stimulation frequency influences mechanical stress measured with TSA.

Since the tested specimens were made out of aluminum, the thermal conductivity is very high. Thus, when the cyclic load was being applied, the temperature increases and decreases. If a variation of the load is slow, the high conductivity of the aluminum may help to dissipate the localized increase of temperature. For the higher frequencies, the material conductivity has less capacity to transfer heat and an increase in the local temperature is observed. This is also observed when observing the temperature images frame by frame. The TSA numerical simulations may provide a better comprehension of experimental observations.

\section{Conclusion}

Higher frequencies resulted in higher mechanical stresses, as the experimental results of thermoelastic stress tests have shown. One may say that the correlation between the maximum stress and the frequency is very high.

For the tested frequencies, a stabilization of the peak stress values for the tested frequencies was not achieved. For this, frequencies of approximately $200 \mathrm{~Hz}$ might be required. In parallel, numeric simulations might also help to comprehend these findings and increase the reliability and accuracy of the TSA in industrial everyday maintenance operations.

\section{REFERENCES}

[1] F. C. Campbell, elements of metallurgy and engineering alloys. ASM International, USA, 2008.

[2] Y. Cheng, Y. Deng, J. Cao, X. Xiong, L. Bai, and Z. Li, "Multi-Wave and Hybrid Imaging Techniques: A New Direction for Nondestructive Testing and Structural Health Monitoring," Sensors, vol. 13, no. 12, pp. 1614616190, 2013.

[3] D. S. Mountain and J. M. B. Webber, "Stress Pattern Analysis By Thermal Emission (SPATE)," 1979, vol. 0164, pp. 189-196.

[4] R. E. R. Eann A. Patterson Richard J. Greene, springer handbook of experimental solid mechanics. springer, 2008. 